\title{
LAS POLITICAS CURRICULARES EN EL ÁREA DE LENGUAJE Y LA EVALUACIÓN DE LA ESCRITURA EN EL MARCO DE UN CONCURSO
}

\author{
LANGUAGE CONTENT AREA CURRICULUM POLICIES AND WRITTEN ASSESSMENT IN A CONTEST
}

\author{
Carlos A. Castrillón ${ }^{1}$, Graciela Uribe Álvarez ${ }^{2}$, Miguel Ángel Caro Lopera ${ }^{3}$
}

Fecha de recibido: Enero 20 de 2011

Fecha de aceptado: Junio 12 de 2011

Correspondencia: Programa de Español y Literatura. Universidad del Quindío.Av. Bolivar calle 12 norte Armenia Quindío Colombia.

Profesor del Programa de Español y Literatura. Correo electrónico: sonorilo@yahoo.com

Profesora del Programa de Español y Literatura. Correo electrónico: gracielauribeal@yahoo.com

${ }^{3}$ Profesor del Programa de Español y Literatura. Correo electrónico: maincaro@yahoo.com

\section{RESUMEN}

En este artículo se contrastan los resultados del proyecto de investigación, "Análisis de una muestra representativa de los relatos presentados al Concurso Nacional de Cuento Gabriel García Márquez (2007)", con tres referentes importantes: La Revolución Educativa, que prevé las metas de desarrollo de la educación colombiana; los Lineamientos y Estándares del área de Lenguaje, que proporcionan el marco de trabajo para los docentes, y la evaluación masiva por competencias (pruebas SABER). Este ejercicio permite comprobar la distancia que existe entre lo deseable y el desempeño real de los estudiantes en la producción de textos escritos.

PALABRAS CLAVE: Competencias en lenguaje, evaluación masiva, políticas curriculares, producción escrita.

\begin{abstract}
In this paper, the results of the research project "Representative Analysis of Stories from Gabriel García Márquez National Story Contest (2007)" are contrasted with three important topics: Educational Revolution; which determines the development of Colombian's educational goals along with guidelines and standards for the Spanish language area, which in turn provides a framework to the educators, and finally a massive evaluation of the education's competencies (pruebas SABER). This research allows us to compare the gap between the student's expected scores level and the real results achieved by the students at the time of producing written texts.
\end{abstract}

KEY WORDS: Language Competencies, Massive Evaluation, Currículum Policies, Written Production.

\section{INTRODUCCIÓN}

Desde la promulgación de la Ley General de Educación en 1994, y con la paulatina puesta en marcha de los mecanismos de evaluación y control que allí se previeron, la educación colombiana ha venido sufriendo cambios sustanciales que comprometen la labor docente. Estos cambios implican una nueva forma de concebir el ejercicio pedagógico y la responsabilidad por los resultados del mismo, que ahora son objeto de control mediante lineamientos, estándares y evaluación permanente. La implementación de las políticas de evaluación de la calidad de la educación y de desarrollo curricular ha significado un gran reto en todos los niveles.
En este contexto, el proyecto de investigación Análisis de una muestra representativa de los relatos presentados al Concurso Nacional de Cuento Gabriel García Márquez, desarrollado por convenio entre el Grupo de Investigación Dilema de la Universidad del Quindío y el Ministerio de Educación Nacional (2008), se propuso estudiar los textos presentados a dicho evento, con el fin de generar hipótesis en relación con los procesos de la escritura en la escuela y de establecer líneas para programas de formación que permitieran cualificar las prácticas de los docentes. El proyecto, pensado desde los modelos de Van Dijk $(1978,1983)$ y De Beaugrande y Dressler (1972), produjo los siguientes documentos: "Hallazgos teóricos, metodológicos y prácticos para la enseñanza

Rev. Invest. Univ. Quindío (22): 9- 19. Armenia - Colombia 
de la producción de textos escritos", "Contraste de los resultados del análisis con las políticas educativas y la evaluación por competencias", "Recomendaciones para la definición y evaluación de programas de formación inicial y en servicio de docentes del área de lenguaje", "Propuestas dirigidas a los establecimientos educativos para cualificar los procesos de desarrollo de competencias comunicativas en los estudiantes" y "Estados del arte de las concepciones sobre prácticas de lectura y escritura en la Educación Básica, Media y Superior colombiana".

A continuación se propone una mirada contrastada entre los resultados del proyecto y las metas de esas políticas, a partir del segundo documento mencionado. Para el efecto, determinamos los desempeños que, según estos resultados, se convierten en facilitadores o en obstáculos para el logro de los objetivos en el desarrollo de las competencias asociadas a la producción de textos escritos. Los referentes para la discusión sobre la distancia que existe entre lo deseable y el desempeño real de los estudiantes en la producción de textos escritos son: La Revolución Educativa, que prevé las metas de desarrollo de la educación colombiana; los Lineamientos y Estándares del área de Lenguaje, que proporcionan el marco de trabajo para los docentes, y los soportes conceptuales de la evaluación masiva (pruebas SABER).

El proyecto que sirve de base para la reflexión se estructuró desde un enfoque mixto que imbricaba los métodos cuantitativo y cualitativo. Desde el primero, se ponderaron los aspectos más recurrentes que se desprenden de la muestra analizada, como fruto de la aplicación a cada texto de una grilla evaluativa de 50 desempeños; y desde el segundo, se prestó atención a relaciones pertinentes que, sin necesidad de abundantes respaldos numéricos, aportaran por sí solas argumentos significativos a la generación de hipótesis. Teniendo en cuenta que la muestra está constituida por cuentos que fueron enviados a un concurso literario, los 50 desempeños se organizaron en dos plantillas, una para determinar el rendimiento del componente textual y otra para la valoración de los aspectos propios del texto narrativo (Bal, 1987) y de la comunicación literaria (Mayoral, 1987). La muestra significativa (10\%) se distribuyó, según las categorías que proponía el mismo concurso, de la siguiente manera:

Rev. Invest. Univ. Quindío (22): 9- 19. Armenia - Colombia
Tabla 1. Distribución de la muestra por categorías

\begin{tabular}{lcc}
\hline Categoría & Número de cuentos & Muestra \\
\hline A. De grado1 a grado 7 & 12.390 & 1.239 \\
B. De grado 8 a grado 11 & 12.599 & 1.259 \\
C. Educación Superior & 7.023 & 702 \\
Total de cuentos & 32.012 & 3.202 \\
\hline
\end{tabular}

\section{CONTRASTE DE LOS RESULTADOS DEL ANÁLISIS CON LAS POLÍTICAS CURRICULARES Y LA EVALUACIÓN POR COMPETENCIAS}

\section{Políticas educativas: Revolución Educativa}

El Plan Sectorial 2006-2010: Revolución Educativa, que recoge las metas y estrategias gubernamentales para el desarrollo de la educación colombiana, formula cinco temas fundamentales, de los cuales uno, "calidad de la educación", se relaciona directamente con los resultados de esta investigación. Nos referimos a la evaluación permanente del sistema mediante pruebas nacionales e internacionales, con el propósito de generar las acciones que garanticen que los estudiantes alcancen niveles de desempeño que impliquen la posibilidad de proyección en el mundo laboral y académico. Al respecto, se manifiesta que "uno de los indicadores más importantes del estado de la calidad de la educación es el desempeño de los estudiantes en pruebas que miden el nivel de desarrollo de competencias durante su paso por el sistema educativo: competencias básicas, ciudadanas y laborales" (MEN, 2008: 24). Dentro de las competencias básicas están las relacionadas con el lenguaje, específicamente la comprensión lectora y, más recientemente, la producción escrita, tal como se define en los marcos conceptuales de la prueba SABER. Los diagnósticos generados avalan la pertinencia de los planes de mejoramiento que elaboran las instituciones educativas y los procesos de control por parte del Estado.

Para el logro de sus metas, la política de calidad propone articular todos los niveles alrededor del trabajo por competencias: "El desarrollo de las competencias básicas y ciudadanas está en el centro del quehacer educativo, desde la educación inicial hasta la superior, y constituye el núcleo común de los currículos en todos los niveles" (MEN, 2008: 29). Esto se evidencia en sus cuatro estrategias: consolidación del sistema de aseguramiento de la calidad de la educación para todo el sistema, implementación de programas para el desarrollo de competencias, perfeccionamiento profesional de docentes y directivos y fomento de la investigación. 
Lo encontrado en la muestra estudiada hace suponer que las metas trazadas en el marco de la Revolución Educativa se están cumpliendo en lo relacionado con la oralidad, mientras que se evidencian problemas preocupantes en los desempeños que sustentan el proceso de producción de un texto escrito. Esto puede explicarse por el hecho de que la evaluación por competencias ha profundizado en la comprensión lectora, generando una presión a la que los maestros han respondido; no ocurre lo mismo con la escritura, que sólo recientemente el MEN se ha propuesto evaluar de modo sistemático.

\section{Políticas Curriculares implicadas}

El documento que define el marco general de la profesión docente especifica los saberes básicos y las competencias que deben orientar la formación del maestro. Esos saberes y competencias se pueden resumir así (Salinas y Forero, 2004: 23):

- Saber qué es, cómo se procesa y para qué el énfasis. Debe conocer la estructura epistemológica de las disciplinas a la luz de los Lineamientos y Estándares Curriculares.

- Saber enseñar el énfasis. Se trata de transformar los saberes disciplinares en saberes enseñables.

- Saber organizar, desarrollar y dirigir situaciones y ambientes de aprendizaje. El planteamiento de los procesos de enseñanza, de aprendizaje y de evaluación, lo que significa concebir, diseñar, ejecutar espacios, dispositivos y procedimientos para la enseñanza.

- Saber evaluar. Se refiere a los saberes relacionados con las distintas prácticas evaluativas: institucionales -aula, proyectos educativos- y externas (SABER, ECAES).

- Saber proponer, desarrollar, sistematizar y evaluar proyectos educativos y de aula. Esta competencia tiene como propósito identificar y resolver problemas educativos y escolares institucionales, que respondan a las necesidades del contexto.

- Saber articular la práctica pedagógica a los contextos. Se trata de relacionar la práctica pedagógica con lo institucional, lo administrativo y lo político; pertinencia con el contexto; articulación con el PEl e identidad.

En este conjunto de exigencias, que comprometen tanto la formación inicial como el ejercicio profesional, el maestro debe estar atento a los llamados del contexto y a la responsabilidad consciente y proactiva para el logro de su función social. Para esto es indispensable el conocimiento y la asunción de los principios y propósitos de las políticas curriculares, que se exponen en los Lineamientos Curriculares y los Estándares Básicos.

\section{Lineamientos Curriculares de Lengua Castellana}

Tabla 2. Procesos para la escritura, según los Lineamientos Curriculares.

\begin{tabular}{|c|c|c|c|}
\hline Nivel & Componente & Se ocupa de & Que se entiende como \\
\hline \multirow[t]{4}{*}{ Intratextual } & Semántico & Microestructuras & $\begin{array}{l}\text { - Estructura de las oraciones y relaciones entre } \\
\text { ellas. } \\
\text { - Coherencia local. } \\
\text { - Coherencia lineal y cohesión. }\end{array}$ \\
\hline & & Macroestructuras & $\begin{array}{l}\text { - Coherencia global. } \\
\text { - Seguimiento de un eje temático a lo largo del } \\
\text { texto. }\end{array}$ \\
\hline & Sintáctico & Superestructuras & $\begin{array}{l}\text { - Forma global como se organizan los } \\
\text { componentes de un texto. El esquema lógico de } \\
\text { organización del texto. }\end{array}$ \\
\hline & & Léxico & $\begin{array}{l}\text { - Campos semánticos. Universos coherentes de } \\
\text { significación. Tecnolectos. Léxicos particulares. } \\
\text { - Coherencia semántica. Usos particulares de } \\
\text { términos (regionales, técnicos...). }\end{array}$ \\
\hline Intertextual & Relacional & $\begin{array}{l}\text { Relaciones } \\
\text { con otros textos }\end{array}$ & $\begin{array}{l}\text { - Contenidos o informaciones presentes en un } \\
\text { texto que provienen de otro. Citas literales. } \\
\text { Fuentes. Formas, estructuras, estilos tomados de } \\
\text { otros autores, o de otras épocas. Referencias a } \\
\text { otras épocas, otras culturas. }\end{array}$ \\
\hline Extratextual & Pragmático & Contexto & $\begin{array}{l}\text { - Contexto, entendido como la situación de } \\
\text { comunicación en la que se dan los actos de habla. } \\
\text { Intención y componentes ideológico y político } \\
\text { presentes en un texto. } \\
\text { - Usos sociales de los textos en contextos de } \\
\text { comunicación, reconocimiento del interlocutor, } \\
\text { selección de un léxico particular o un registro } \\
\text { lingüístico: Coherencia pragmática. }\end{array}$ \\
\hline
\end{tabular}




\section{- 12 - Políticas Curriculares y evaluación de escritura en el área de Lenguaje}

Los Lineamientos Curriculares de Lengua Castellana recuperan la reflexión sobre los diferentes elementos que conforman un texto, entendido este como "un tejido de significados que obedece a reglas estructurales semánticas, sintácticas y pragmáticas" (MEN, 1998: 61). Así, para la producción de textos escritos, el documento propone pensar en tres tipos de procesos (Tabla 2).

Los procesos del nivel intratextual tienen que ver con "estructuras semánticas y sintácticas, presencia de microestructuras y macroestructuras, lo mismo que el manejo de léxicos particulares y de estrategias que garantizan coherencia y cohesión a los mismos: cuantificadores, conectores, pronominalización, marcas temporales y marcas espaciales" (MEN, 1998: 61-62); los del nivel intertextual se basan en las habilidades que necesita desarrollar el estudiante para reconocer las relaciones existentes entre el texto y otros textos; y los del nivel extratextual pertenecen al orden de lo pragmático y buscan reconstruir "el contexto o situación de comunicación en que se producen o aparecen los textos, con el componente ideológico y político que subyace a los mismos y con el uso social que se hace de ellos".

Tabla 3. La producción de textos en los Lineamientos Curriculares y los resultados del proyecto

\begin{tabular}{|c|c|c|c|}
\hline \multicolumn{2}{|c|}{ Nivel } & \multicolumn{2}{|c|}{ Comportamiento de los desempeños } \\
\hline & & Facilitadores & Obstáculos \\
\hline \multirow{4}{*}{ 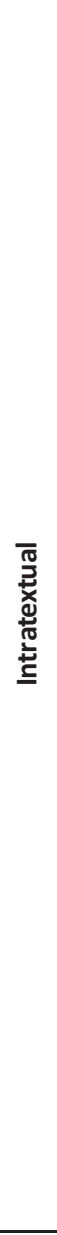 } & 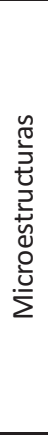 & $\begin{array}{l}\text { Aceptable en la construcción de } \\
\text { oraciones simples. } \\
\text { Sobresaliente en concordancias sujeto- } \\
\text { verbo. } \\
\text { Sobresaliente en concordancias } \\
\text { nombre-determinante. } \\
\text { Sobresaliente en concordancias } \\
\text { nombre-deíctico en el nivel intrafrásico. } \\
\text { Sobresaliente en concordancias } \\
\text { nombre-deíctico en el nivel interfrásico. } \\
\text { Sobresaliente en el manejo de } \\
\text { conectores en el nivel intrafrásico. }\end{array}$ & $\begin{array}{l}\text { Muy bajo en el control de erratas. } \\
\text { Muy bajo en la construcción de ora ciones } \\
\text { subordinadas. } \\
\text { Muy bajo en el empleo de signos de } \\
\text { puntuación en los niveles intrafrásico e } \\
\text { interfrásico. } \\
\text { Muy bajo en el empleo de signos de } \\
\text { puntuación en el nivel interfrásico. } \\
\text { Bajo en el control de la repetición } \\
\text { injustificada de palabras. } \\
\text { Bajo en el uso de conectores apropiados } \\
\text { en el nivel interfrásico. }\end{array}$ \\
\hline & $\begin{array}{l}\dot{d} \\
\frac{0}{2} \\
\sum^{\pi}\end{array}$ & $\begin{array}{l}\text { Sobresaliente en el ofrecimiento de } \\
\text { índices textuales que evidencian } \\
\text { coherencia. } \\
\text { Sobresaliente en la elección de un } \\
\text { título significativo. }\end{array}$ & $\begin{array}{l}\text { Bajo en la segmentación del texto en } \\
\text { párrafos que respondan a unidades de } \\
\text { sentido. }\end{array}$ \\
\hline & 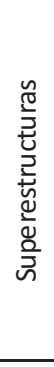 & $\begin{array}{l}\text { Sobresaliente en la conservación de un } \\
\text { esquema narrativo posible. } \\
\text { Sobresaliente en la narración de una } \\
\text { historia completa. } \\
\text { Sobresaliente en el seguimiento de una } \\
\text { secuencia de acciones. } \\
\text { Sobresaliente en la organización de una } \\
\text { estructura temporo-espacial. } \\
\text { Sobresaliente en el desarrollo de una } \\
\text { instancia narracional. }\end{array}$ & $\begin{array}{l}\text { Muy bajo en la propuesta de estructuras } \\
\text { narrativas com plejas. }\end{array}$ \\
\hline & 䒝 & $\begin{array}{l}\text { Sobresaliente en la elección de lexemas } \\
\text { adecuados para lo que quiere expresar. } \\
\text { Sobresaliente en el ofrecimiento de un } \\
\text { contexto para el significado de las } \\
\text { palabras. } \\
\text { Sobresaliente en el conocimiento de los } \\
\text { sentidos de las palabras. }\end{array}$ & \\
\hline 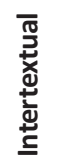 & $\begin{array}{l}\frac{\overline{0}}{\underline{U}} \\
\frac{\pi}{\mathscr{Q}} \\
\simeq\end{array}$ & $\begin{array}{l}\text { Aceptable en el uso de intertextos. } \\
\text { Aceptable en la pertinencia de los } \\
\text { intertextos. }\end{array}$ & \\
\hline
\end{tabular}


Luego de cruzar los niveles que proponen los Lineamientos con los desempeños que en esta investigación se rastrearon, percibimos cierta correspondencia entre unos y otros. La Tabla 3 muestra la contribución de los resultados de la investigación a los niveles y componentes que proponen los Lineamientos para la producción de textos escritos en el área de Lenguaje.

A simple vista se percibe que son más los desempeños que acercan las prácticas de los estudiantes a lo que esperan los Lineamientos Curriculares. Fortalezas inobjetables se aprecian en el proceso intratextual en cuanto a lo superestructural y lexical, mientras que los desempeños relacionados con la micro y la macroestructura se agrupan en iguales porcentajes como facilitadores u obstáculos, lo que fortalece la conclusión a la que llegamos en la investigación acerca de la primacía de lo superestructural sobre lo textual, de la coherencia sobre la cohesión; en palabras del proyecto, la intención narrativa prima sobre el trabajo textual propiamente dicho. En cuanto al nivel intertextual, las fortalezas son también notables, lo que de alguna manera da razones para que no se descuide la enseñanza de la literatura y se defienda su preeminencia como discurso de discursos.

Tal como se había anotado en las conclusiones de la investigación (Camargo, Uribe, Caro y Castrillón, 2008), es claro que los procesos deben fortalecerse irremediablemente en lo extratextual, en cuanto a los aspectos de más difícil dominio: la introducción consciente de rupturas con la norma para configurar una intención estética, la búsqueda de interlocución genuina, la expresión de visiones particulares del mundo y la mirada dialógica. Sin duda, estos aspectos no invocan solamente la consolidación del proceso escritural, sino también la revisión al tipo de lecturas que circulan en la escuela; sólo el acercamiento a estructuras narrativas cada vez más complejas, a las rupturas con la norma y al dialogismo textual propiciará imágenes mentales que más tarde incidan en la creación.

\section{Estándares Básicos de Competencias en Lenguaje}

Concebidos como "criterios claros y públicos que permiten juzgar si un estudiante, una institución o el sistema educativo en su conjunto cumplen con unas expectativas comunes de calidad", los Estándares
Básicos de Competencias en Lenguaje se agrupan en cinco categorías (producción textual, comprensión e interpretación textual, literatura, medios de comunicación y otros sistemas simbólicos, y ética de la comunicación). Su organización se inspira en el enfoque interdisciplinario y funcional-comunicativo de los Lineamientos y supone para cada categoría enunciados identificadores y subprocesos que guardan coherencia horizontal y vertical en función del proceso ininterrumpido de desarrollo de las competencias en lenguaje.

Para el caso concreto de la producción textual, tanto los enunciados identificadores como los subprocesos aumentan en complejidad a medida que se asciende por los grupos de grados. La Tabla 4 da cuenta de tales elementos y muestra la contribución de los resultados de la investigación (desempeños de los estudiantes) a la verificación de la puesta en práctica de los Estándares en el trabajo de aula.

Una mirada somera a los subprocesos revela claras coincidencias con lo que se demostró en el proyecto. Allí partíamos de la concepción de texto, desde la perspectiva de De Beaugrande y Dressler (1972), como acontecimiento comunicativo, por lo cual reconocíamos en él la respuesta a un pacto entre autor y lector en el marco de una cultura y al abrigo de unas condiciones específicas de enunciación. Sosteníamos que los estudiantes cumplían las primeras cláusulas, pero que olvidaban la letra menuda del contrato, lo que, como en cualquier pacto, acarrea la nulidad de la transacción.

En efecto, el primer subproceso recoge fundamentalmente aciertos que se pueden equiparar con el cumplimiento exitoso de una consigna, originada en una convocatoria abierta y clara, que supone al productor del discurso la aceptación de ciertas normas de participación y la respuesta a un rol definido como autor de un cuento que será leído por otros y que aspira a la publicación y al reconocimiento social y económico. Tal éxito obedece no sólo a una estrategia de difusión entre medios de comunicación y escuela, sino también como algo digno de reconocerse- al impulso del docente, admitido como la primera fuente de motivación para la participación en el evento (40\%). La confluencia favorable de todos estos factores puede explicar el abundante número de textos que en la muestra dan cuenta de una intención narrativa ( $98 \%$ en la categoría A; $95 \%$ en la B y $96 \%$ en la C). 
Al primer éxito se suma el de los subprocesos segundo, tercero y cuarto. Lo prueban los buenos desempeños en la conservación de un esquema narrativo posible, la narración de una historia completa, el seguimiento de una secuencia completa de acciones que forman una trama, el desarrollo de una instancia narracional bien definida y la organización de una estructura temporoespacial. Efectivamente, como se reiteraba en el proyecto, a lo largo de las diversas categorías podía advertirse el suficiente grado de rendimiento como para poder declarar que los participantes entendieron la consigna y se dispusieron (como tantos lo expresaron en su título) a contar un cuento.

Ahora bien, en los subprocesos quinto y sexto los problemas empiezan a aflorar. En el marco de exigencias de la práctica de la escritura no bastan las intenciones iniciales, la consigna general, los propósitos de entrada, la intuición del modo de organización del discurso, las líneas generales de un argumento, las palabras para expresarlo. El compromiso que adquiere el autor implica también la corrección paciente, la revisión crítica a todo nivel.

Tabla 4. La producción de textos en los Estándares y los resultados de la investigación.

\begin{tabular}{|c|c|c|}
\hline \multirow[t]{2}{*}{ Subprocesos } & \multicolumn{2}{|c|}{ Comportamiento de los desempeños } \\
\hline & Facilitadores & Obstáculos \\
\hline $\begin{array}{l}\text { 1. Defino una temática para } \\
\text { la producción de un texto } \\
\text { narrativo (posible lector, } \\
\text { propósito comunicativo, } \\
\text { exigencias del contexto y } \\
\text { características de esa } \\
\text { tipología). }\end{array}$ & $\begin{array}{l}\text { - Sobresaliente en la presentación de } \\
\text { una clara intención narrativa. } \\
\text { - Sobresaliente en la elección de } \\
\text { lexemas adecuados para lo que } \\
\text { quiere expresar. } \\
\text { - Sobresaliente en el ofrecimiento de } \\
\text { un contexto para el significado de sus } \\
\text { palabras. } \\
\text { - Sobresaliente en el conocimiento } \\
\text { de los sentidos de las palabras. }\end{array}$ & \\
\hline $\begin{array}{l}\text { 2. Llevo a cabo } \\
\text { procedimientos de búsqueda, } \\
\text { selección y almacenamiento } \\
\text { de información acerca de la } \\
\text { temática que voy a tratar en } \\
\text { mi texto narrativo. }\end{array}$ & $\begin{array}{l}\text { - Sobresaliente en la conservación de } \\
\text { un esquema narrativo posible. }\end{array}$ & \\
\hline $\begin{array}{l}\text { 3. Elaboro un plan textual, } \\
\text { organizando la información } \\
\text { en secuencias lógicas. }\end{array}$ & $\begin{array}{l}\text { - Sobresaliente en la narración de } \\
\text { una historia completa. }\end{array}$ & \\
\hline $\begin{array}{l}\text { 4. Produzco una primera } \\
\text { versión del texto narrativo } \\
\text { teniendo en cuenta } \\
\text { personajes, espacio, tiempos } \\
\text { y vínculos con otros textos y } \\
\text { con mi entorno. }\end{array}$ & $\begin{array}{l}\text { - Sobresaliente en el seguimiento de } \\
\text { una secuencia de acciones. } \\
\text { - Sobresaliente en el desarrollo de } \\
\text { una instancia narracional. } \\
\text { - Sobresaliente en la organización de } \\
\text { una estructura temporo-espacial. }\end{array}$ & \\
\hline $\begin{array}{l}\text { 5. Reviso, socializo y corrijo } \\
\text { mis escritos, teniendo en } \\
\text { cuenta las propuestas de mis } \\
\text { compañeros y profesor, y } \\
\text { atendiendo algunos aspectos } \\
\text { gramaticales (concordancia, } \\
\text { tiempos verbales, } \\
\text { pronombres) y ortográficos } \\
\text { (acentuación, mayúsculas, } \\
\text { signos de puntuación) de la } \\
\text { lengua castellana. }\end{array}$ & $\begin{array}{l}\text { - Sobresaliente en la realización de } \\
\text { operaciones de revisión estructural } \\
\text { del texto. } \\
\text { - Sobresaliente en concordancias } \\
\text { nombre-deíctico en el nivel } \\
\text { intrafrásico. } \\
\text { - Sobresaliente en concordancias } \\
\text { nombre-deíctico en el nivel } \\
\text { interfrásico }\end{array}$ & $\begin{array}{l}\text { - Muy bajo en el empleo correcto de las } \\
\text { mayúsculas. } \\
\text { - Muy bajo en el control de erratas. } \\
\text { - Muy bajo en la construcción de } \\
\text { oraciones subordinadas. } \\
\text { - Muy bajo en el empleo de signos de } \\
\text { puntuación en los niveles intrafrásico e } \\
\text { interfrásico. } \\
\text { - Bajo en la identificación de la } \\
\text { ortografía de las palabras. } \\
\text { - Bajo en la identificación del acento } \\
\text { ortográfico y diacrítico. } \\
\text { - Bajo en el control de la repetición } \\
\text { injustificada de palabras. } \\
\text { - Bajo en la segmentación del texto en } \\
\text { párrafos que respondan a unidades de } \\
\text { sentido. } \\
\text { - Bajo en el uso de conectores } \\
\text { apropiados en el nivel interfrásico. }\end{array}$ \\
\hline $\begin{array}{l}\text { 6. Reescribo el texto a partir } \\
\text { de las propuestas de } \\
\text { corrección formuladas por } \\
\text { mis compañeros y por mí. }\end{array}$ & & $\begin{array}{l}\text { - Muy bajo en la propuesta de miradas } \\
\text { dialógicas al mundo. } \\
\text { - Bajo en el manejo de las estructuras } \\
\text { del texto dialogal. } \\
\text { - Bajo en la expresión de visiones } \\
\text { particulares del mundo. }\end{array}$ \\
\hline
\end{tabular}

Rev. Invest. Univ. Quindío (22): 9- 19. Armenia - Colombia 
Tabla 5. Relación entre los criterios de la prueba SABER y los resultados del proyecto.

\begin{tabular}{|c|c|c|}
\hline Categorías & Criterios en SABER & Comportamiento de los desempeños \\
\hline Legibilidad & $\begin{array}{l}\text { - Legibilidad del escrito. } \\
\text { - Control del uso de mayúsculas y } \\
\text { minúsculas. }\end{array}$ & $\begin{array}{l}\text { - Sobresaliente en el cuidado de } \\
\text { detalles estéticos para la legibilidad de } \\
\text { su texto. } \\
\text { - Muy bajo en el empleo correcto de } \\
\text { las mayúsculas. } \\
\text { - Muy bajo en el control de erratas. }\end{array}$ \\
\hline $\begin{array}{l}\text { Control formal } \\
\text { del léxico }\end{array}$ & $\begin{array}{l}\text { - Control en la utilización del léxico. } \\
\text { - Conocimiento de la forma de los } \\
\text { lexemas. }\end{array}$ & $\begin{array}{l}\text { - Aceptable control de la unión y } \\
\text { separación inapropiada de lexemas. }\end{array}$ \\
\hline $\begin{array}{l}\text { Conexión y } \\
\text { conectores }\end{array}$ & $\begin{array}{l}\text { - Control de los recursos de conexión a } \\
\text { nivel intrafrásico, interfrásico y entre } \\
\text { unidades mayores. }\end{array}$ & $\begin{array}{l}\text { - Sobresaliente en el manejo de } \\
\text { conectores en el nivel intrafrásico. } \\
\text { - Bajo en el uso de conectores } \\
\text { apropiados en el nivel interfrásico. }\end{array}$ \\
\hline $\begin{array}{l}\text { Cohesión y } \\
\text { co-referencias }\end{array}$ & $\begin{array}{l}\text { - Control de los recursos de cohesión a } \\
\text { nivel intrafrásico, interfrásico y entre } \\
\text { unidades mayores }\end{array}$ & $\begin{array}{l}\text { - Sobresaliente en el seguimiento de } \\
\text { concordancias nombre-deíctico en los } \\
\text { niveles intrafrásico e interfrásico. }\end{array}$ \\
\hline Puntuación & $\begin{array}{l}\text { - Uso de signos de puntuación con } \\
\text { función textual entre frases y entre } \\
\text { párrafos. }\end{array}$ & $\begin{array}{l}\text { - Muy bajo en el empleo de la } \\
\text { puntuación en los niveles intrafrásico e } \\
\text { interfrásico. }\end{array}$ \\
\hline $\begin{array}{l}\text { Estructura } \\
\text { textual }\end{array}$ & $\begin{array}{l}\text { - Superestructura (secuencia) textual. } \\
\text { - Cierre de la superestructura. } \\
\text { - La superestructura coincide con el tipo } \\
\text { textual demandado en el enunciado. }\end{array}$ & $\begin{array}{l}\text { - Sobresaliente en el ofrecimiento de } \\
\text { índices que evidencian coherencia. } \\
\text { - Sobresaliente en la conservación de } \\
\text { un esquema narrativo posible. } \\
\text { - Sobresaliente en la narración de una } \\
\text { historia completa. } \\
\text { - Sobresaliente en el seguimiento de } \\
\text { una secuencia de acciones. } \\
\text { - Sobresaliente en el desarrollo de una } \\
\text { instancia narracional. } \\
\text { - Sobresaliente en la organización de } \\
\text { una estructura temporo-espacial. } \\
\text { - Sobresaliente en las operaciones de } \\
\text { revisión estructural del texto. }\end{array}$ \\
\hline $\begin{array}{l}\text { Ortografía } \\
\text { y aspectos } \\
\text { formales }\end{array}$ & - Control de los recursos. & $\begin{array}{l}\text { - Bajo en la identificación de la } \\
\text { ortografía de las palabras. } \\
\text { - Bajo en la identificación del acento } \\
\text { ortográfico y diacrítico. }\end{array}$ \\
\hline Intencionalidad & $\begin{array}{l}\text { - Pertinencia respecto de la solicitud del } \\
\text { enunciado (tema y tipo textual } \\
\text { demandado). } \\
\text { - Se anticipa el auditorio. }\end{array}$ & $\begin{array}{l}\text { - Sobresaliente en la presentación de } \\
\text { una clara intención narrativa. }\end{array}$ \\
\hline Tipo Textual & $\begin{array}{l}\text { - El tipo textual seleccionado atiende a la } \\
\text { petición del enunciado. }\end{array}$ & \\
\hline Léxico & $\begin{array}{l}\text { - Uso de un léxico coloquial (cotidiano). } \\
\text { - Uso de un léxico que oscila entre lo } \\
\text { coloquial y lo formal. } \\
\text { - Uso y control de un léxico formal. }\end{array}$ & $\begin{array}{l}\text { - Sobresaliente en la elección de } \\
\text { lexemas adecuados para lo que quiere } \\
\text { expresar. } \\
\text { - Sobresaliente en el ofrecimiento de } \\
\text { un contexto para el significado de las } \\
\text { palabras. } \\
\text { - Sobresaliente en el conocimiento de } \\
\text { los sentidos de las palabras. } \\
\text { - Bajo en el control de la repetición } \\
\text { injustificada de palabras. }\end{array}$ \\
\hline $\begin{array}{l}\text { Reconocimiento } \\
\text { de la práctica } \\
\text { sociodiscursiva }\end{array}$ & $\begin{array}{l}\text { - Se reconoce el auditorio a quien se } \\
\text { dirige el texto. } \\
\text { - Control del léxico, el estilo y el modo } \\
\text { de tratar el tema, según la práctica socio- } \\
\text { discursiva que demanda el enunciado. }\end{array}$ & $\begin{array}{l}\text { - Muy bajo en la búsqueda de } \\
\text { interlocución genuina. }\end{array}$ \\
\hline $\begin{array}{l}\text { Punto de vista } \\
\text { (tesis) }\end{array}$ & $\begin{array}{l}\text { - Se enuncia y desar rolla un punto de } \\
\text { vista. }\end{array}$ & $\begin{array}{l}\text { - Bajo en la expresión de visiones } \\
\text { particulares del mundo. } \\
\text { - Muy bajo en la propuesta de miradas } \\
\text { dialógicas al mundo. }\end{array}$ \\
\hline
\end{tabular}

Rev. Invest. Univ. Quindío (22): 9- 19. Armenia - Colombia 
Como puede verse en la columna de factores obstaculizadores, a esta fase final del proceso de escritura no llegaron la gran mayoría de los participantes.

\section{Evaluación de la escritura en las pruebas SABER}

La evaluación masiva en lenguaje se fundamenta en los niveles crecientes de comprensión lectora, no en los procesos de producción escrita. Esto se debe a que la escritura es más difícil de evaluar que la lectura, a tal punto que varias pruebas no la contemplan (PISA, Examen de Estado) y otras, que la han integrado, no ofrecen resultados analíticos (SERCE) o no se conocen (LLECE). Además, no existe suficiente información histórica relacionada con la evaluación sistemática de la producción escrita. El problema es mucho más difícil de abordar en la escritura creativa, como es el caso del corpus de estudio (cuentos presentados a un concurso literario). El análisis de una producción creativa no puede proceder con la misma exactitud estadística y jerárquica, pues depende de la valoración de un lector que debe distinguir las débiles diferencias entre lo textual y lo literario. En consecuencia, los resultados del proyecto no son transferibles ni equiparables; se trata sólo de una aproximación bajo el supuesto de que los procesos asociados a la lectura y a la escritura están relacionados de algún modo en el desarrollo de las competencias.

Las pruebas SABER y el Examen de Estado comparten el mismo soporte conceptual en su última formulación (Castillo et al., 2007b) y producen resultados análogos; ambas evaluaciones están centradas en el análisis de las competencias textual y discursiva, entendidas como "el conjunto de posibilidades que tiene un estudiante para comprender, interpretar, organizar y producir actos de significación a través de distintos sistemas de signos lingüísticos y no lingüísticos". Esto remite a un campo muy amplio y diverso en el que se encuentran la comprensión lectora y la producción escrita como formas de "saber-saber-hacer" con el lenguaje. Los presupuestos que explican lo deseable para la producción escrita en las pruebas SABER coinciden con las tendencias internacionales:

Producir un texto, es entonces, construir un escenario, sus actores y relaciones, sus posibles espectadores y críticos a través del discurso. En este sentido el trabajo sobre los procesos de coherencia y cohesión presuponen un saber previo de estrategias de organización textual y discursiva que se movilizan en el acto mismo de la escritura: construcciones sintácticas particulares, posibles recorridos temáticos, tipificación de enunciadores y narradores, continuidad y apertura de los contenidos temáticos, modelos textuales que permitan inferir o presuponer intenciones comunicativas; un corpus de situaciones y relaciones entre sujetos que generan mundos posibles, etc.(MEN, 2004).

Lo que se privilegia aquí no es la corrección gramatical, la ortografía y la sujeción a las formalidades de la lengua, que son por supuesto importantes, sino la capacidad para significar en el proceso de escritura de un texto. A partir de los presupuestos de la Lingüística Textual, lo que se busca es valorar el desarrollo de un tema, el manejo de las estructuras pertinentes y el cierre estructural. La evaluación de textos escritos, que se basa en la pregunta abierta pero motivada se convierte en fundamental si se desea dar cuenta de un modo más completo de las competencias en lenguaje. A pesar de la dificultad implícita en este tipo de evaluación, derivada de la imposibilidad de calificarla de modo expedito y de la dificultad de establecer parámetros valorativos, se suele proceder a un muestreo que se valora de modo cualitativo, según una tabla de rasgos textuales que cobijan la mayoría de los factores que influyen en la producción escrita.

El proceso de producción escrita es complejo por las estrategias que debe utilizar quien construye el texto para organizar sus ideas en una unidad coherente y con sentido, y se relaciona con la configuración del otro o posible lector y el planteamiento de una intención comunicativa. El estudiante debe responder interrogantes como: ¿a quién le estoy escribiendo?, ¿para qué?, ¿cómo hacerlo? A través de una pregunta abierta, se le pide al estudiante construir un texto que obedezca a unas instrucciones específicas en relación con un tema determinado, generalmente derivado de la prueba misma, de situaciones hipotéticas o de otros textos. Se indaga por la manera como se construye sentido atendiendo a una superestructura o a la conservación de un tópico.

Tomemos como referencia la aplicación 2005-2006 de las pruebas SABER. Para esa oportunidad se trató de valorar el desempeño en la interpretación y producción de textos escritos, en el marco de las competencias 
textual y discursiva y en situaciones concretas: los estudiantes de grados 5ㅇ y 9으, "como lectores o productores de tipos de texto que habitualmente circulan en la escuela y la sociedad" (Castillo et al., 2007b: 17b). En el caso de la escritura, la evaluación verifica los mecanismos de textualización y puesta en discurso y la ubicación en una práctica socio-cultural, todo según los Lineamientos Curriculares y los Estándares Básicos. Aunque esos resultados no son equiparables con los del proyecto sobre textos narrativos, porque no se puede comparar un producto de la actividad creadora con un escrito en ambiente de evaluación y porque las metodologías de valoración son diferentes, es posible hacer un ejercicio sobre las tendencias que muestran los estudiantes a la hora de asumir la producción escrita. La interpretación de los resultados (Castillo et al., 2007), nos permite advertir las siguientes conclusiones:

1. La escritura de un texto de estructura compleja presenta problemas cuando es el estudiante el que debe elegir las estrategias discursivas adecuadas.

2. Un gran porcentaje de estudiantes no alcanza a concebir un texto que tenga en cuenta al lector ("escribir un texto para otro") mediante estrategias claras que impliquen un interlocutor o destinatario.

3. Los desempeños relacionados con la sintaxis del texto (conexión, cohesión) son aceptables, en general, y aumentan con el grado de escolaridad.

Ahora bien, se pueden contrastar estas conclusiones generales con las que derivan del estudio para los aspectos textuales, dejando de lado las consideraciones propiamente literarias. La Tabla 5 muestra las categorías y desempeños de la propuesta de evaluación de la producción escrita en la prueba SABER (Castillo et al., 2007b), que corresponden al dominio textual (conexión, cohesión, puntuación, progresión temática, ortografía), al dominio discursivo (tipo de texto, intencionalidad y léxico) y a la práctica sociocultural, en relación con los hallazgos del análisis de los cuentos presentados al concurso.

Como puede verse, los criterios pertinentes encontraron correlato entre los desempeños contemplados en la plantilla diseñada para el proyecto. Tales congruencias permitirían a futuro, cuando los análisis de la escritura en las pruebas SABER se acoplen al modelo completo, comparar producciones escritas gestadas en diferentes condiciones de enunciación, lo que podría acercar las interpretaciones obtenidas a través de estos métodos a la realidad de las aulas colombianas.

De todos modos, la Tabla 5 muestra los puntos débiles de la producción escrita de los estudiantes colombianos, tal como hemos evidenciado en este estudio, y permite prever las acciones necesarias para abordar esos problemas desde estrategias globales y trabajo de aula.

\section{CONCLUSIÓN}

Por lo general, las evaluaciones y documentos discutidos son consistentes y reflejan el punto de vista contemporáneo, según el cual los estudiantes deberían ser capaces de elaborar y ampliar el significado de lo que leen y escriben en función de una práctica sociodiscursiva. El problema para contrastar los resultados consiste, más bien, en la falta de información histórica y sistemática sobre la evaluación de la producción escrita, a lo que se suma el hecho problemático de la escritura creativa (cuentos presentados a un concurso literario), para la que se dificulta establecer categorías de valoración.

Sin embargo, haciendo abstracción de lo anterior, y mirados los cuentos como textos en el marco de dicha práctica sociodiscursiva, es posible afirmar que el espíritu de los Lineamientos Curriculares de Lengua Castellana y de los Estándares Básicos de Competencias en Lenguaje, claramente emparentado con un enfoque funcional comunicativo de la lengua, desde el estudio de tipologías textuales y de géneros discursivos, dista mucho de materializarse en los desempeños estudiantiles, tal como pudimos verificar en el análisis de la muestra de cuentos del concurso. Quienes deben transponer la visión de las políticas curriculares y hacerlas efectivas en el aula, los maestros, no enfrentan el asunto desde la conceptualización, sino desde modelos iterativos que funcionan al estilo de recetas.

El análisis de los resultados de las pruebas de evaluación por competencias en relación con el lenguaje, los factores asociados y la exigencia de los nuevos marcos legales para la educación, evidencian 
una situación crítica en las aulas y un desconocimiento de las teorías contemporáneas sobre comprensión y producción textual. Se hace necesario, entonces, que los docentes se apropien de los conceptos vertidos en los documentos de política curricular y de las nuevas teorías en relación con la enseñanza y el aprendizaje de la lengua materna, como sustento frente a las actuales realidades educativas, al igual que de las nuevas estrategias de enseñanza y aprendizaje relacionadas con la comprensión y producción textual. Para esto parece provechoso revisar las diferentes propuestas para la evaluación del proceso de producción de textos, en especial las derivadas de la amplia reflexión sobre el modelo SERCE.
La comparación de los resultados de esta investigación con las políticas curriculares y la evaluación por competencias demuestra que, a pesar de la importancia que se da al tema en todos los ámbitos de la educación y la presencia cotidiana de la lectura y la escritura en la escuela, en lo que respecta a la escritura ese interés no va de la mano de estrategias claras, pues en la práctica de aula se enfatiza más en el producto (el texto escrito) que en el proceso: la escritura como sucesión de borradores que, en virtud de una intención y una práctica discursiva, se revisan, reelaboran y corrigen, como manifestación de la voluntad de entrar en el juego del intercambio discursivo.

\section{BIBLIOGRAFÍA}

Atorresi, A. (2005). Competencias para la vida en las evaluaciones de lectura y escritura (SERCE - LLECE). Buenos Aires: OREALC.

Bal, M. (1987). Teoría de la narrativa. Madrid: Cátedra.

Bogoya, J. D. et al. (2005). Segundo Estudio Regional Comparativo y Explicativo (SERCE). Análisis Curricular. Santiago de Chile: OREALC/UNESCO.

Camargo, Z. y Castrillón, C. A. (2006). "Diseño de un desarrollo curricular para el área de Lenguaje desde los conceptos de género discursivo y tipología textual". Cuadernos Interdisciplinarios Pedagógicos, Congreso Internacional en Investigación Educativa y Didácticas, Universidad del Quindío, (7): 133-144.

Camargo, Z., Caro, M. A. y Uribe, G. (2009). Didáctica de la comprensión y producción de textos académicos. Armenia: Universidad del Quindío.

Camargo, Z., Uribe, G., Caro, M. A. y Castrillón, C. A. (2008). Análisis de una muestra representativa de los relatos presentados al Concurso Nacional de Cuento Gabriel García Márquez (2007). Informe Ejecutivo. Armenia: Universidad del Quindío. Disponible en http://www.ascun.org.co/eventos/IIICNC/Analisis de los relatos/Analisis de los relatos presentados al Concurso Nacional de Cuento 2007.pdf

Caro, M. A. y Castrillón, C. A. (2008). "La comprensión de lectura en los estudiantes de grado 5o del Municipio de Armenia: Análisis de resultados de un simulacro de Pruebas Saber". Cuadernos Interdisciplinarios Pedagógicos, Facultad de Educación, Universidad del Quindío, (9): 11-20.

Castillo, M. J. et al (2007). SABER, área de Lenguaje. Análisis de preguntas, aplicación 2005-2006. Bogotá: ICFES.

Castillo, M. J. et al (2007b). Sobre las pruebas SABER y de Estado: Una mirada a su fundamentación y orientación de los instrumentos en Lenguaje. Bogotá: ICFES.

Castrillón, C. A. (2005). "La competencia comunicativa en los alumnos de educación básica del municipio de Armenia". Cuadernos Interdisciplinarios Pedagógicos, Facultad de Educación, Universidad del Quindío, (6): 55-64.

De Beaugrande, R. \& Dressler, W. (1972). Introducción a la lingüística del texto. Trad. S. Bonilla. Barcelona: Ariel, 1997. 
Grupo Didactext (2003). "Modelo sociocognitivo, pragmalingüístico y didáctico para la producción de textos escritos". Didáctica (Lengua y Literatura), (15): 77-104. Madrid: Universidad Complutense.

ICFES (2007). Colombia en PISA 2006. Bogotá.

ICFES (2008). PISA 2009, guía de orientación. Prueba piloto Colombia. Bogotá.

Jurado, F. (2000). "Lenguaje, Competencias Comunicativas y Didáctica: Un estado de la cuestión". Seminario Nacional sobre la investigación educativa y pedagógica en Colombia. Balance de una época. Colciencias, Socolpe e ICFES.

Jurado, F. y Bustamante, G. (Comps). (1993). Los procesos de la escritura. Bogotá: Magisterio.

Laboratorio Latinoamericano de Evaluación de la Calidad de la Educación (2008). Los aprendizajes de los estudiantes de América Latina y el Caribe. Primer reporte de los resultados del Segundo Estudio Regional Comparativo y Explicativo. Santiago de Chile: LLECE, UNESCO.

Mayoral, J. A. (Comp.) (1987). Pragmática de la comunicación literaria. Madrid: Arco/Libros.

MEN (1998). Lengua Castellana. Lineamientos Curriculares. Bogotá: Cooperativa Editorial Magisterio.

MEN (2004). SABER: Plan de seguimiento 1992-2005. Bogotá.

MEN (2006). Estándares Básicos de Competencias en Lenguaje, Matemáticas, Ciencias y Ciudadanas. Bogotá.

MEN (2008). Plan Sectorial 2006-2010: Revolución Educativa. Bogotá.

Organización para la Cooperación y el Desarrollo Económico, OCDE (2004). Learning for Tomorrow's World. First Results from PISA 2003. Paris.

Organización para la Cooperación y el Desarrollo Económico, OCDE (2008). Informe PISA 2006. Competencias científicas para el mundo del mañana. Madrid: Santillana.

Pérez Abril, M. (2003). Leer y Escribir en la escuela. Algunos escenarios pedagógicos y didácticos para la reflexión. Bogotá: ICFES, MEN.

Salinas, M. L. y Forero, F. (2004). Marco general de la profesión del maestro. Bogotá: Ascofade-ICFES.

Uribe, G., Castrillón, C. A. y Camargo, Z. (2010). “La escritura de un cuento y el cumplimiento de las cláusulas de un pacto comunicativo". Magis, Universidad Javeriana, 3(5): 125-134.

Valdés, H. et al. (2008). Segundo Estudio Regional Comparativo y Explicativo (SERCE). Los aprendizajes de los estudiantes de América Latina y el Caribe. Santiago de Chile: OREALC/UNESCO.

Van Dijk, T. (1978). Estructuras y funciones del discurso. México: Siglo XXI, 1980.

Van Dijk, T. (1983). La ciencia del texto. Un enfoque interdisciplinario, 4‥ reimp. Trad. S. Hunzinger. Barcelona: Paidós, 1997. 